\title{
Ultra-short-term prediction method of photovoltaic electric field power based on ground-based cloud image segmentation
}

\author{
Runjie Shen ${ }^{1}$, Ruimin Xing ${ }^{1 *}$, Yiying Wang ${ }^{1}$, Danqiong Hua ${ }^{1}$, Ming $\mathrm{Ma}^{1,2}$ \\ ${ }^{1}$ College of Electronic and Information Engineering, Tongji University, Shanghai, 201800, China \\ ${ }^{2}$ Electric Power Research Institute, State Grid Gansu Electric Power Company, Lanzhou, Gansu, 730000, China
}

\begin{abstract}
As a large number of photovoltaic power stations are built and put into operation, the total amount of photovoltaic power generation accounts for an increasing proportion of the total electricity. The inability to accurately predict solar energy output has brought great uncertainty to the grid. Therefore, predicting the future power of photovoltaic fields is of great significance. According to different time scales, predictions are divided into long-term, medium-term and ultra-short-term predictions. The main difficulty of ultra-short-term forecasting lies in the power fluctuations caused by sudden and drastic changes in environmental factors. The shading of clouds is directly related to the irradiance received on the surface of the photovoltaic panel, which has become the main factor affecting the fluctuation of photovoltaic power generation. Therefore, sky images captured by conventional cameras installed near solar panels can be used to analyze cloud characteristics and improve the accuracy of ultra-short-term predictions. This paper uses historical power information of photovoltaic power plants and cloud image data, combined with machine learning methods, to provide ultra-short-term predictions of the power generation of photovoltaic power plants. First, the random forest method is used to use historical power generation data to establish a single time series prediction model to predict ultra-short-term power generation. Compared with the continuous model, the root mean square (RMSE) error of prediction is reduced by $28.38 \%$. Secondly, the Unet network is used to segment the cloud image, and the cloud amount information is analyzed and input into the random forest prediction model to obtain the bivariate prediction model. The experimental results prove that, based on the cloud amount information contained in the cloud chart, the bivariate prediction model has an $11.56 \%$ increase in prediction accuracy compared with the single time series prediction model, and an increase of $36.66 \%$ compared with the continuous model.
\end{abstract}

\section{Introduction}

The output power of photovoltaic panels mainly depends on the total solar radiation incident on the panel. The variability of solar energy resources and the uncertainty associated with forecasting are the reasons behind most of the issues that must be addressed to maintain grid stability. Although in theory, the theoretical irradiance calculation value can be obtained through the rotation and translation of the earth relative to the sun. However, there are unexpected changes in the amount of solar radiation reaching the surface of the earth. The main reason for this comes from the existence of clouds, which randomly block the sun's rays and give a certain degree of uncertainty to the prediction of photovoltaic power generation.

The strong randomness, intermittentness and uncontrollability of photovoltaic power generation lead to considerable fluctuations in photovoltaic output, which makes the safe and reliable operation of the power grid extremely challenging and restricts the development of photovoltaic power generation. Accurate and effective photovoltaic power generation power prediction can reduce the negative impact of photovoltaic uncertainty. Photovoltaic power prediction methods can generally be divided into physical methods [2], [3], statistical methods [4]-[7], artificial intelligence (AI) methods [8]-[14] and other hybrid methods. Generally, artificial intelligence methods include artificial neural networks (ANN) [9], support vector machines (SVM) [10], [11 and extreme learning machines (ELM) [12], [13], random forests [14]. In [10], V-SVM is used, Cuckoo search algorithm is used to optimize parameters to predict wind speed, and grey relational analysis is used to determine the input set. In [13], stacked ELM is used to predict wind speed, which is an advanced ELM algorithm under the deep learning framework. In [14], random forest is used as a supervised prediction model, and the data-driven dimensionality reduction process and weighted voting method are used to optimize the random forest algorithm in the training process and prediction process.

Installing a sky imager in the photovoltaic field can obtain real-time cloud images above the field, thereby

*Corresponding author's e-mail: xrm_tju@163.com 
monitoring the thickness and movement of the clouds in the sky. Inputting the information contained in the cloud chart into the prediction model can improve the prediction accuracy. Literature [15] began to use sky images from two Total Sky Imagers (TSI) located in large power plants. The method proposed by the author works in a time range of 30 seconds to 15 minutes in a 30 -second interval, generating a cloud image and a power histogram normalized by the expected clear sky power output. Then the clear and turbid modes are used to assign the value of the normalized power to the shaded and non-shaded cells of the cloud image. Therefore, the complete power output of the photovoltaic plant can be explained. However, this approach leads to poor performance compared to persistence. An average RMSE higher than $40 \%$ was obtained on each of the two test days. In [16], the author made a comparison between technologies (cloud tracking, ARMA and k-NN), and applied ANN to re-predict. This optimization will input lagging behind the generated value (return to 30 minutes in a 5-minute interval) and the prediction of the baseline model. The results showed that cloud tracking technology (as described above) performed the worst, with prediction skills of $-71.3 \%$ and $-18.9 \%$ in the prediction range of 5 minutes and 15 minutes, respectively. They found two reasons for this poor performance: the two-dimensional processing of clouds, simplifying their 3D nature, and misrecognizing cloudy/clear pixels, especially near the sun. However, when re-forecasting was performed, the method performed better than ARMA (predictive skills of 12.9$19.4 \%$ ), k-NN (predictive skills of $-2.66 \%$ to $0.3 \%$ ), and persistence, showing prediction skills of $15.1 \%$ And $26.2 \%$. Each is a 15 -minute field of view. If there is no re-prediction, the best model is ARMA, with a prediction skill of up to $8 \%$.
This paper proposes a scenario with a single data, that is, only power and cloud image data, and hopes to autonomously extract cloud amount information from cloud images through a deep network (without cloud tracking, 3D modeling, etc.) to help improve prediction accuracy.

The main research contents are as follows:

1. Based on the time series information of a single power, a random forest rolling prediction model is established to perform ultra-short-term prediction of the output power of the photovoltaic field

2. Use Unet network to segment the cloud image to obtain cloud amount information

3. Based on cloud cover information and power data, a bivariate random forest rolling prediction model is established.

\section{Method}

The workflow of the ultra-short-term forecasting method proposed in this research is shown in Figure 1 and Figure2, which mainly include univariate time series model and bivariate time series model. The univariate time series model uses the power history data vector to make rolling forecasts. The bivariate prediction model includes two parts: cloud image processing and historical power data. The main steps include the segmentation of the ground-based cloud image and the calculation of cloud amount. The unet deep network is used to encode and decode the cloud image to obtain the divided cloud image, and then calculate the cloud amount on this basis. Through the segmentation and extraction of historical cloud images, the cloud amount historical data vector is obtained, and the cloud amount historical data vector and the power historical data vector are sent to the random forest rolling prediction model to obtain the power prediction value.

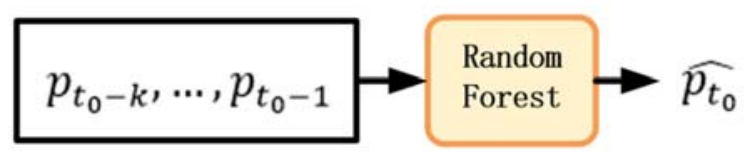

Figure 1. Univariate time series prediction model

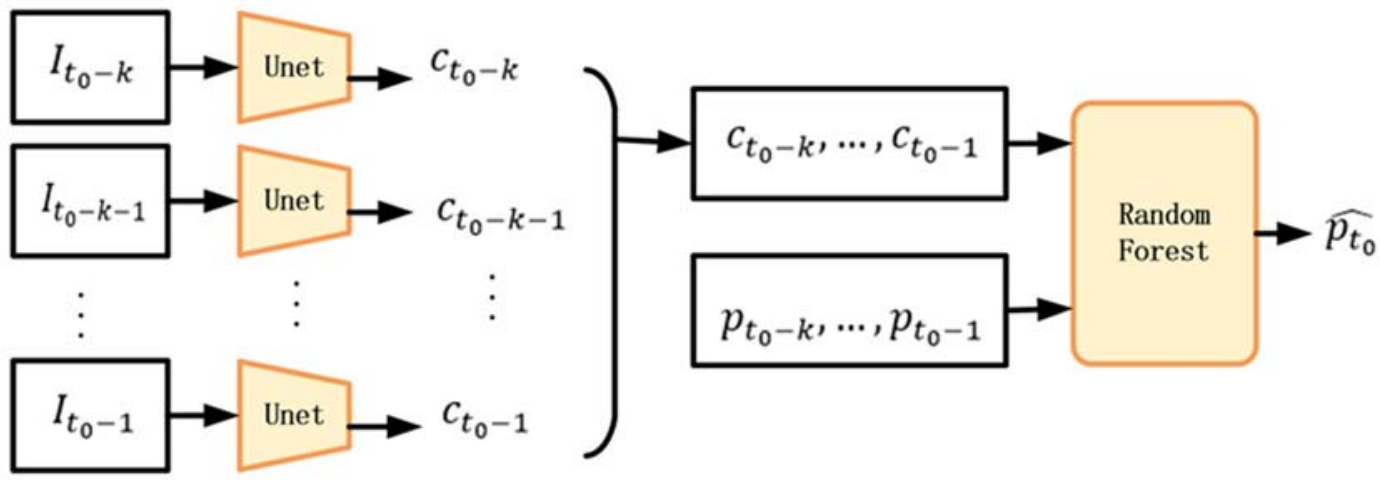

Figure 2. Bivariate time series forecasting model

Among them, $p_{t_{0}}$ represents the real power value at time $t_{0}, \widehat{p_{t_{0}}}$ represents the predicted power value at time $t_{0}, I_{t_{0}}$ represents the cloud image at time $t_{0}$, and $c_{t_{0}}$ represents the cloud cover calculation at time $I_{t_{0}}$ value. 


\subsection{Single time series variable random forest rolling prediction model}

Random forest is an ensemble algorithm (Ensemble Learning), which belongs to the Bagging type. By combining multiple weak classifiers, the final result is voted or averaged, so that the result of the overall model has high accuracy and generalization performance. Random forest has good performance and application in power prediction. The main reason is that it has a certain degree of anti-overfitting ability, so that its algorithm performance is relatively stable in various situations. In the ultra-short-term prediction problem, the input of the random forest prediction model is the vector $p_{t_{0}-k}, \ldots, p_{t_{0}-1}$ composed of historical power data, and the prediction result is $\widehat{p_{t_{0}}}$, which is right Estimated value of power at $t_{0}$ time. Due to the calculation speed, the rolling prediction method is adopted. As shown in Figure 3, one month's data is used to predict the photovoltaic power in the next 20 minutes, and the database is updated every 10 minutes (adding the most recent measured value and suggesting the farthest Measured value), then make a prediction for the next 20 minutes.

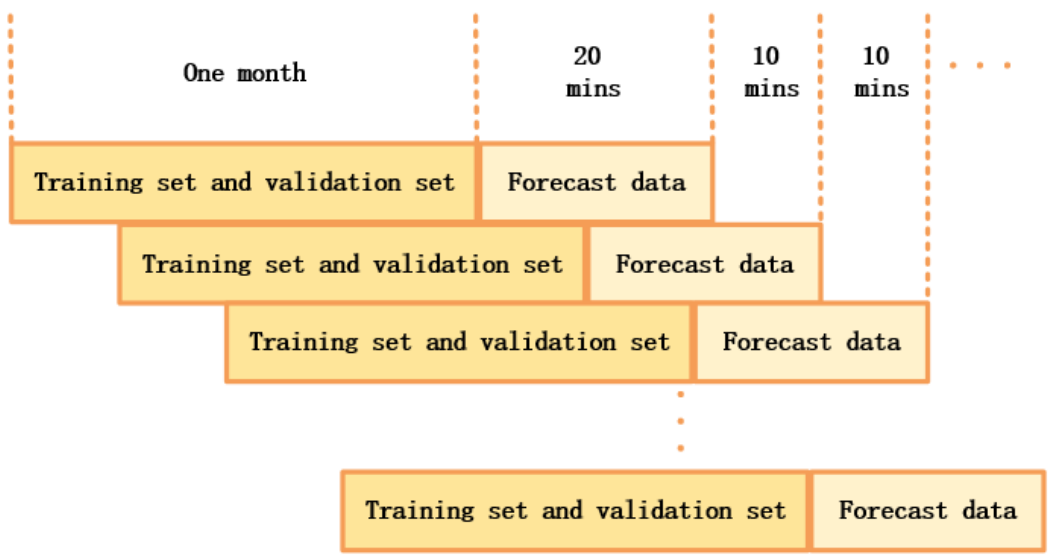

Figure 3. Schematic diagram of rolling forecasting method

\subsection{Unet network segmentation cloud image}

Although Unet [17] is a model born in 2015, it is still the most widely used model in current semantic segmentation projects. Many of the top LB players on kaggle use this model. Compared with the traditional semantic segmentation network FCN, Unet also adopts an encoding-reverse encoding structure. The major structural changes are in the up-sampling stage, and the up-sampling layer also includes many layers of features. Another advantage over FCN is that Unet only needs one training session, while FCN needs three training sessions. When Unet first appeared, it was applied to the semantic segmentation of biomedical images. The characteristic is that compared with the ordinary deep network that requires thousands of annotated training samples, literature [17] proposes an image enhancement method, which effectively expands the available data samples, so that Unet no longer needs thousands of data samples at every turn. This network can be trained end-to-end from very few images and is superior to the previous best method in the ISBI challenge (sliding window convolutional network) for segmentation of neuron structures in electron microscope stacks. Using the same network trained on transmitted light microscope images (Phase Contrast and DIC), in these categories, it drastically won the 2015 ISBI Cell Tracking Challenge. In addition, the network is fast. On recent GPUs, $512 \times 512$ image segmentation takes less than one second [17].

In this paper, the Unet network is used to segment the cloud image, so as to segment and identify the cloud area in the cloud image, and obtain relevant information such as cloud amount. The supervised learning method with labels is adopted to judge the cloud image pixel by pixel as a cloud area. The result of cloud image segmentation is shown in Figure 4:

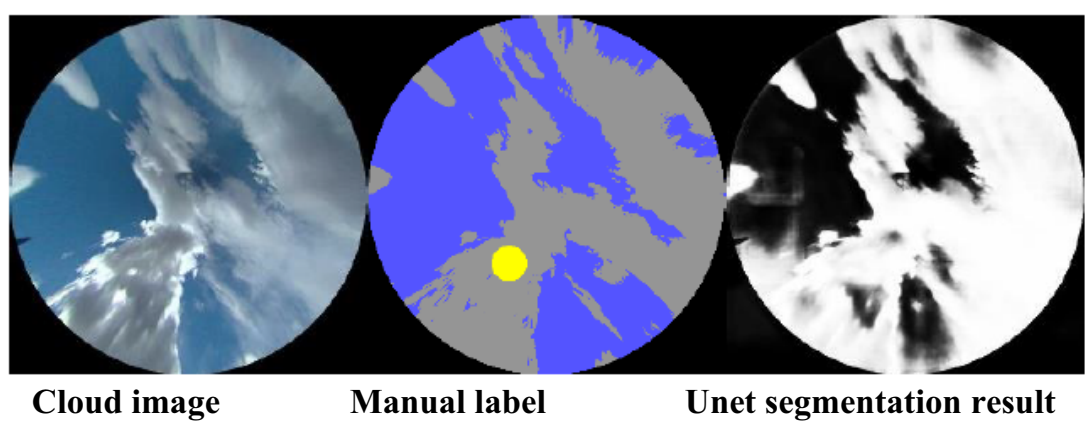

Figure 4. Cloud image segmentation results 


\subsection{Cloud computing and bivariate time series prediction model}

The result of cloud image segmentation is a gray-scale image, and formula 1 is used to calculate cloud amount information

$$
\alpha=\frac{N_{\text {cloud }}}{N_{\text {total }}}
$$

Among them, $N_{\text {cloud }}$ represents the number of pixels judged to be clouds, $N_{\text {cloud }}$ represents the total number of pixels in the cloud image, and $\alpha$ represents the cloud amount detection result at the current moment.

Using the above formula, the historical cloud image can be processed and calculated to obtain the historical cloud amount time series data. Figure 5 is the cloud amount data obtained after processing the cloud image on a certain day.

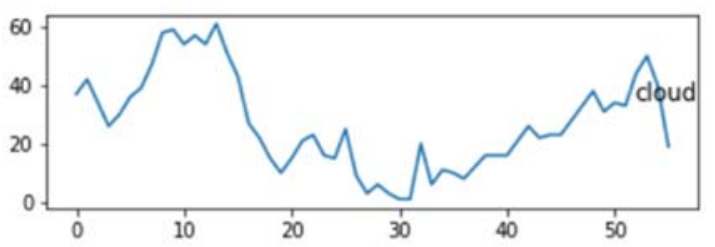

Figure 5. Cloud cover time series data

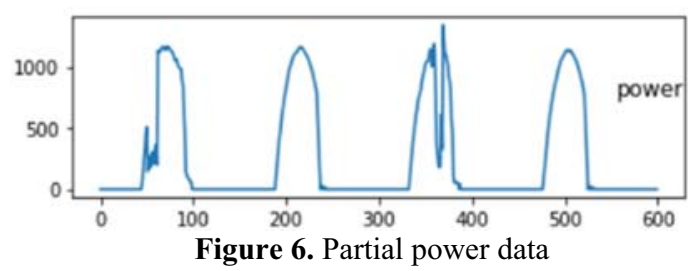

\section{Experimental results}

\subsection{Experimental data}

The data used in this article comes from the National Renewable Energy Laboratory[18]. Including cloud image data from 7:30 on January 1, 2018 to 16:50 on December 1, 2018, with a time interval of one shot every ten minutes; and power data for the whole year of 2018, with a measurement interval of ten minutes.

\subsection{Single time series forecast model results}

Using the power data, the prediction interval is 20 minutes, the prediction frequency is every 10 minutes, and the prediction range is an ultra-short-term prediction of 7 days. The root mean square error (RMSE) is used to predict the accuracy, and the root mean square error is calculated using formula 2 .

$$
\mathrm{RMSE}=\sqrt{\frac{1}{N} \sum_{i=1}^{N}\left(\widehat{p}_{\imath}-p_{i}\right)^{2}}
$$

Where $\mathrm{N}$ is the number of predicted time points, $\widehat{p}_{\imath}$ represents the predicted power value at the i-th time point, and $p_{i}$ represents the real measured value at the ith time point. In order to measure the advancement of the prediction model, a continuous model is used as a control. Persistent models are often used as benchmarks for more developed models because they are the simplest. The model assumes that the conditions (irradiance, power output, cloud cover, etc.) remain constant between $\mathrm{t}$ and $\mathrm{t}+1$. The "naive persistence model" assumes that the predicted power for the time range will be the same as the last value measured. For example, for an ultra-short-term prediction task at 20minute intervals, the power of 7:30 will be the same as the power of 7:10. In the ultra-short-term time series data prediction that this article faces, the continuous model prediction result is shown in Figure 7, RMSE $=236.5173$. Figure 8 shows the results of a single time series prediction model, RMSE $=169.3987$.

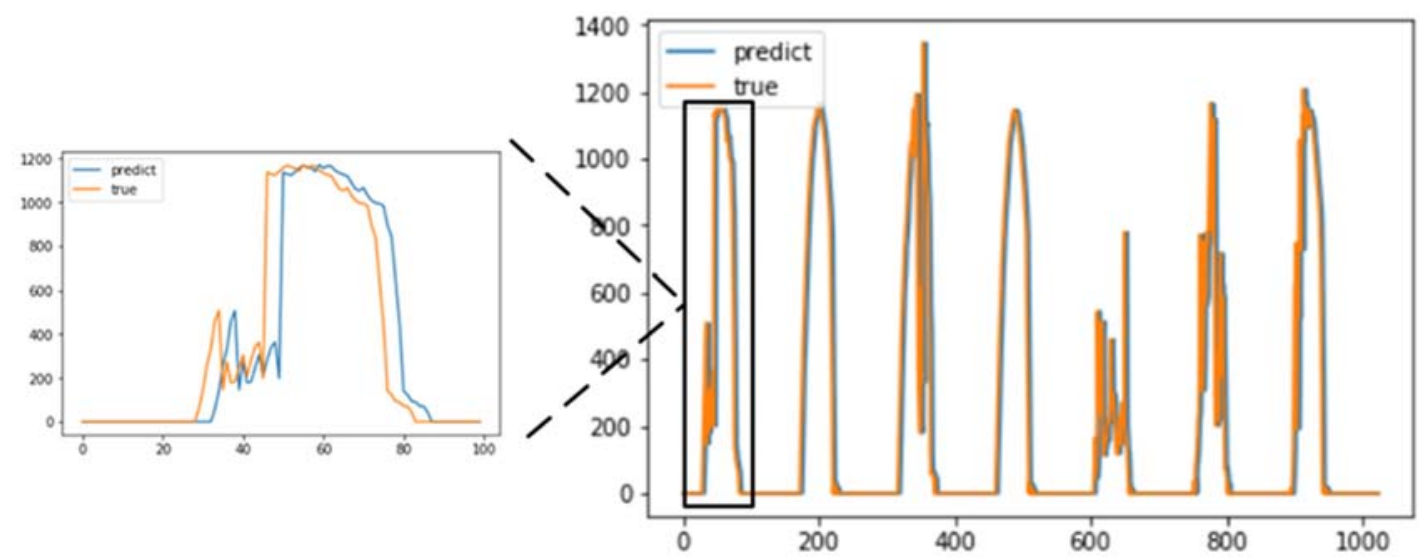

Figure 7. Continuous model prediction results. 


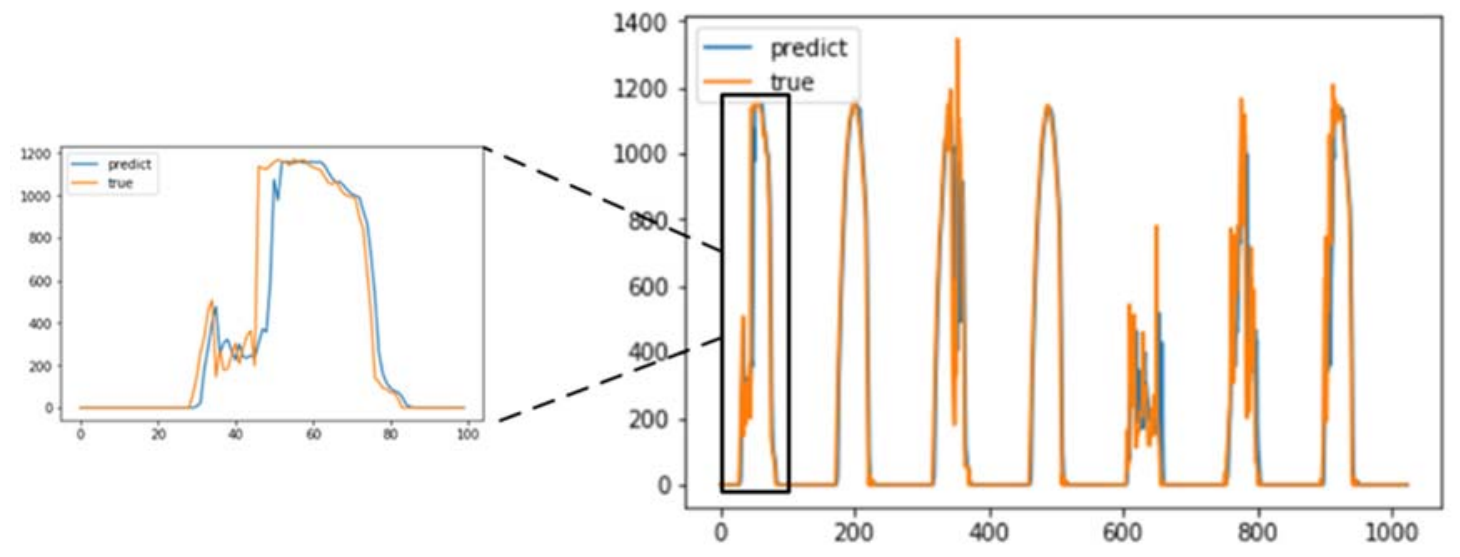

Figure 8. Single time series forecast model forecast results.

\subsection{Cloud image segmentation results and cloud amount calculation}

Select a few representative cloud images of cloudy, less cloud, and thin cloud weather conditions. The segmentation results and cloud amount calculations are shown in Table 1:

Table 1. Cloud image segmentation results.

\begin{tabular}{|c|c|c|c|c|}
\hline Date & Clouo image & Manual label & segmentation results & Cloud amount \\
\hline $\begin{array}{l}\text { January } \\
8, \quad 2018 \\
9: 20\end{array}$ & & & & $98.6 \%$ \\
\hline $\begin{array}{l}\text { January } \\
5, \quad 2018 \\
16: 30\end{array}$ & & & & $91.5 \%$ \\
\hline $\begin{array}{l}\text { January } \\
3, \quad 2018 \\
12: 40\end{array}$ & & & & $46.3 \%$ \\
\hline $\begin{array}{l}\text { January } \\
5, \quad 2018 \\
14: 00\end{array}$ & & & & $52.6 \%$ \\
\hline
\end{tabular}




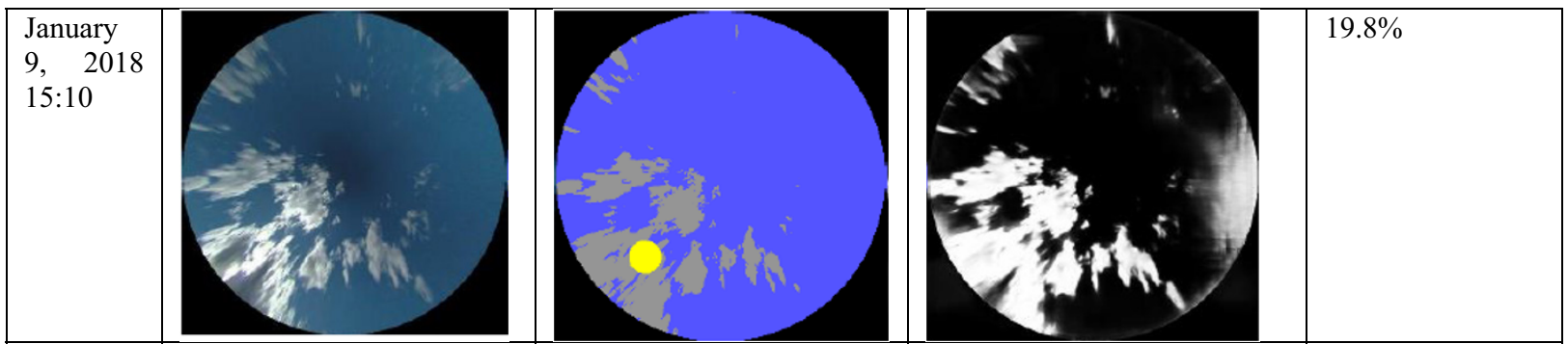

\subsection{Bivariate time series data prediction model}

the prediction accuracy is improved by $36.66 \%$. Compared with the single time series prediction model, the prediction accuracy is improved by $11.56 \%$.

The dual-variable time series data prediction model $\mathrm{RMSE}=149.8202$. Compared with the continuous model,

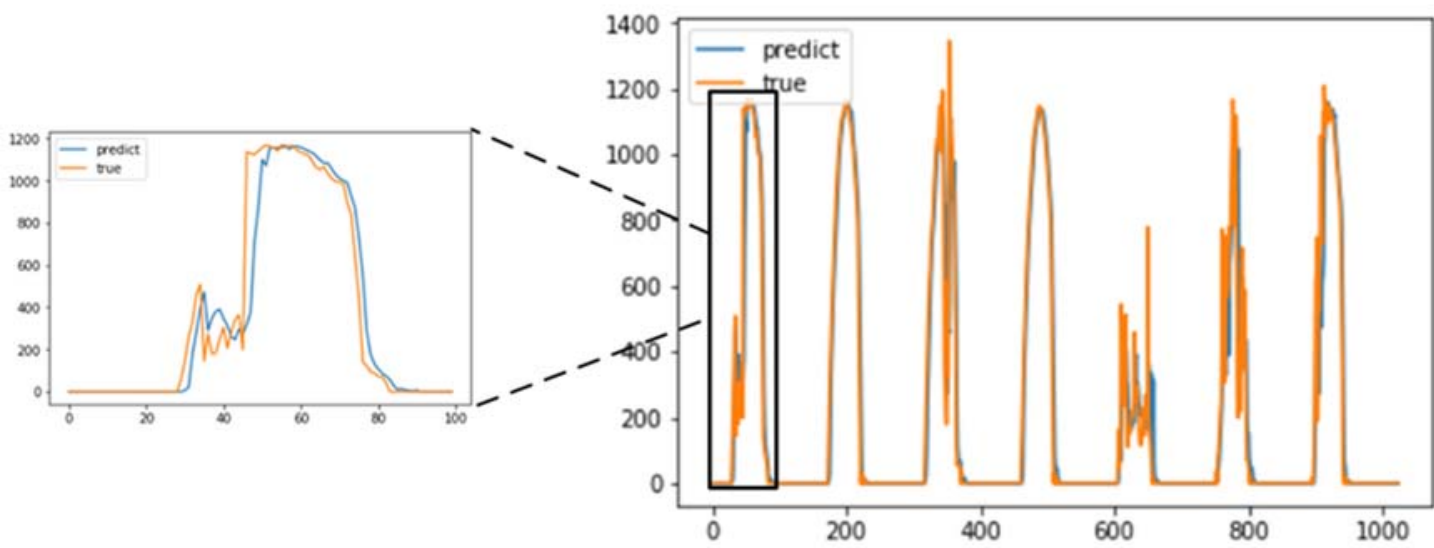

Figure 9. Bivariate prediction model prediction results

\section{Conclusion}

In this research, a time series prediction model based on Unet network segmentation cloud image is proposed. After the Unet network encodes and decodes the groundbased cloud image, the cloud amount information can be extracted. The result proves that the cloud image segmentation result of Unet network is similar to manual labeling, which solves the problem of cloud location in cloud image and enables cloud image information to be quickly converted into cloud amount information. In comparison with the continuous model and the single time series model, the prediction accuracy of the bivariate forecast model considering cloud amount information has been significantly improved, which confirms the results of cloud image segmentation from the side. This study provides a simple scenario for the ultra-short-term prediction of photovoltaic electric fields, that is, only power data and cloud image data; and in this scenario, it provides a reasonable use of cloud images to improve prediction accuracy.

\section{References}

1. Antonanzas J, Osorio N, Escobar R, et al. Review of photovoltaic power forecasting[J]. Solar Energy, 2016, 136: 78-111.

2. W. Zhang, Z. Qu, K. Zhang, W. Mao, Y. Ma, X. Fan,
"A combined model based on CEEMDAN and modified flower pollination algorithm for wind speed forecasting", Energy Convers. Manage., vol. 136, pp. 439-451, Mar. 2017.

3. J. Zhao, Z.-H. Guo, Z.-Y. Su, Z.-Y. Zhao, X. Xiao, F. Liu, "An improved multi-step forecasting model based on WRF ensembles and creative fuzzy systems for wind speed", Appl. Energy, vol. 162, pp. 808-826, Jan. 2016.

4. M. Lydia, S. S. Kumar, A. I. Selvakumar, G. E. P. Kumar, "Linear and non-linear autoregressive models for short-term wind speed forecasting", Energy Convers. Manage., vol. 112, pp. 115-124, Mar. 2016.

5. E. Erdem, J. Shi, "ARMA based approaches for forecasting the tuple of wind speed and direction", Appl. Energy, vol. 88, no. 4, pp. 1405-1414, 2011.

6. R. G. Kavasseri, K. Seetharaman, "Day-ahead wind speed forecasting using f-ARIMA models", Renew. Energy, vol. 34, no. 5, pp. 1388-1393, 2009.

7. Z. Song, Y. Jiang, Z. Zhang, "Short-term wind speed forecasting with Markov-switching model", Appl. Energy, vol. 130, no. 3, pp. 103-112, 2014.

8. S. Baran, "Probabilistic wind speed forecasting using Bayesian model averaging with truncated normal components", Comput. Statist. Data Anal., vol. 75 , no. 3 , pp. $227-238,2014$ 
9. H. Mori, S. Okura, "An ANN-based method for wind speed forecasting with S-transform", Proc. IEEE Region 10 Conf., pp. 642-645, Nov. 2016.

10. P. Jiang, Y. Wang, J. Wang, "Short-term wind speed forecasting using a hybrid model", Energy, vol. 119, pp. 561-577, Jan. 2017.

11. D. Bai, J. He, X. Wang, "Combination model for forecasting wind speed forecasting wind speed based on adaptive PSO-ELM", Acta Energiae Solaris Sinica, vol. 36, no. 3, pp. 792-797, 2015.

12. Z. Yihui et al., "A hybrid short-term wind speed forecasting model based on ensemble empirical mode decomposition and improved extreme learning machine", Power Syst. Protection Control, vol. 10, no. 42, pp. 29-34, Dec. 2017.

13. X. Luo, J. Sun, L. Wang, W. Wang, W. Zhao, J. Wu, J.-H. Wang, Z. Zhang, "Short-term wind speed forecasting via stacked extreme learning machine with generalized correntropy", IEEE Trans. Ind. Informat., vol. 14, no. 11, pp. 4963-4971, Nov. 2018.

14. Z. Sun, H. Sun, J. Zhang, "Multistep wind speed and wind power prediction based on a predictive deep belief network and an optimized random forest", Math. Problems Eng., vol. 7, no. 10, pp. 115, Jul. 2018.

15. Urquhart B, Ghonima M, Nguyen D, et al. Sky imaging systems for short-term forecasting[J]. Solar Energy Forecasting and Resource Assessment, 2013: 195-232.

16. Chu Y, Urquhart B, Gohari S M I, et al. Short-term reforecasting of power output from a 48 MWe solar PV plant[J]. Solar Energy, 2015, 112: 68-77.

17. Ronneberger O, Fischer P, Brox T. U-net: Convolutional networks for biomedical image segmentation[C]//International Conference on Medical image computing and computer-assisted intervention. Springer, Cham, 2015: 234-241.

18. Andreas, A.; Stoffel, T.; (1981). NREL Solar Radiation Research Laboratory (SRRL): Baseline Measurement System (BMS); Golden, Colorado (Data); NREL Report No. DA-550056488.http://dx.doi.org/10.5439/1052221 